\title{
ESTIMATIVA DAS GERAÇÕES ANUAIS DE Gyropsylla spegazziniana (LIZER, 1917) EM FUNÇÃO DE SUA EXIGÊNCIA TÉRMICA
}

\author{
ESTIMATION OF THE ANNUAL GENERATIONS OF Gyropsylla spegazziniana \\ (LIZER, 1917) IN FUNCTION ON THE THERMAL REQUERIMENTS
}

\author{
Luís Antônio Chiaradia $^{1}$ José Maria Milanez $^{2}$ Alexsandro Zidko ${ }^{3}$
}

RESUMO

\begin{abstract}
Gyropsylla spegazziniana (Lizer, 1917) (Hemiptera, Psyllidae) ou "ampola" é uma praga da erva-mate (Ilex paraguariensis) que induz a hipertrofia de folhas novas, para abrigar as ninfas. Folhas com estes sintomas caem após a saída dos insetos, reduzindo a produtividade dos ervais. Para estudar as interferências dos fatores ambientais sobre a flutuação populacional desta praga, foram realizadas amostragens quinzenais, em dois ervais situados em Chapecó-SC, no período de 1997 a 2000. A triagem das amostras foi realizada na Eparri/CPPP e totalizou 21.044 espécimes, com razão sexual de 0,42 . A análise de correlação entre os insetos capturados no período 1998/2000 e a média mensal de temperatura mínima (Tmi) acusou $r=0,63$ e a análise de regressão linear expressou a equação $\hat{y}=-1.621,47+156,37 \mathrm{Tmi}$, com $r^{2}=0,40 . \mathrm{A}$ temperatura base $(\mathrm{Tb})$ de $10,36{ }^{\circ} \mathrm{C}$ foi estimada ao atribuir valor zero para esta equação. A constante térmica $(K)$ de 399,52 grausdia $(G D)$ foi calculada por $K=D(T-T b)$, onde $D=27,29$ dias do ciclo de vida da "ampola" a $25^{\circ} \mathrm{C}(T)$. Os 3.219,30 GD anuais foram obtidos pela média dos somatórios anuais das médias mensais das temperaturas médias, diminuídas da $T b$ e multiplicadas pelo número de dias de cada mês, nos meses que a $T m i>T b$. A divisão do GD anual pela $K$ resultou em 8,05 possíveis gerações anuais desta praga.
\end{abstract}

Palavras-chave: erva-mate, Ilex paraguariensis, insecta, gerações anuais, graus-dia.

\section{SUMMARY}

Gyropsylla spegazziniana (Lizer, 1917) (Hemiptera, Psyllidae) or "ampul" is a pest of paraguay-tea (Ilex paraguariensis) that provoques the growth in the structure of new leaves for ninfal protection. Leaves with these symptoms fall after insects left causing produtivity reduction. To study the environmental factors interference in the population flutuation of

\begin{abstract}
this pest, two orchards were biweekly sampled from 1997 to 2000 in Chapecó, SC. The samples were evaluated in the Agricultural Research Enterprise - Epagri/CPPP, and totaled 21,044 insects with $0.42 \%$ of sexual ratio. Correlation analyses between insects captured from 1998/2000 and monthly mean minimum temperature (Tmi) accused $r=0.63$ and the linear regression analyses showed the equation $\hat{y}=-1,621.47+156.37 \mathrm{Tmi}$, with $r^{2}=0.40$. The basic temperature $(\mathrm{Tb})$ of $10.36^{\circ} \mathrm{C}$ was obtained by giving value zero to this equation. The thermal constant $(K)$ of 399.52 day-degrees $(G D)$ was calculated using $K=D(T-T b)$, in which "D" was 27.29 the days of biological cicle of "ampul", with $25^{\circ} \mathrm{C}(T)$. The 3,219.30 annual GD was calculated with the mean of the total sum of monthly mean medium temperature, lessenig from $\mathrm{Tb}$ and multiplicating number of days, in the months in which Tmi > Tb. The annual GD divided to CT resulted in 8.05 possible annual generations of this insect.
\end{abstract}

Key words: paraguay-tea, Ilex paraguariensis, insecta, annual generations, day-degrees.

\section{INTRODUÇÃO}

A erva-mate Ilex paraguariensis (Aquifoliaceae) é uma essência florestal que ocorre nas regiões temperadas e subtropicais da América do Sul, entre os paralelos $21^{\circ}$ e $30^{\circ}$ de latitude Sul e meridianos $48^{\circ}$ e $56^{\circ}$ de longitude Oeste, principalmente em altitudes que variam entre 500 a 1000m (DA CROCE \& FLOSS, 1999).

A erva-mate é industrializada para preparar a erva do chimarrão, pós-solúveis, chás, refrigerantes e outras bebidas, destinadas ao mercado interno e também para a exportação

\footnotetext{
${ }^{1}$ Engenheiro agrônomo, MSc. em Fitotecnia, Epagri - CPPP, CP 791, telefone: (0xx49) 328-4277, fax: 328-6017, 89801-970, Chapecó, SC. E-mail: chiaradia@epagri.rct-sc.br. Autor para correspondência.

${ }^{2}$ Engenheiro Agrônomo, Ph.D. em Entomologia, Epagri - CPPP. E-mail: milanez@epagri.rct-sc.br.

${ }^{3}$ Graduando em Biologia, Universidade do Oeste de Santa Catarina, Chapecó, SC.
} 
(ANUÁRIO BRASILEIRO DA ERVA-MATE, 2000). O setor ervateiro no Brasil concentra-se em 486 municípios, onde existem aproximadamente 725 indústrias de beneficiamento e envolve direta e indiretamente 710.000 pessoas, constituindo-se num importante segmento agroindustrial da região Sul do País (DA CROCE \& FLOSS, 1999).

A produção brasileira de erva-mate no ano de 1999 foi de $666.000 \mathrm{t}$ de erva verde, que correspondem a 266.664t de produtos industrializados, sendo os Estados do Paraná, de Santa Catarina e do Rio Grande do Sul responsáveis por $42 \%$; $37 \%$ e $21 \%$ da produção, respectivamente (ANUÁRIO BRASILEIRO DA ERVA-MATE 2000).

Por muitos anos, a erva-mate foi extraída de matas e de ervais nativos conduzidos em consórcio com pastagens ou culturas anuais. Mais recentemente, passou a ser cultivada em reflorestamentos puros. Esse sistema facilita o manejo e aumenta a produtividade, mas favorece o ataque de doenças e pragas. Neste sentido, a Gyropsylla spegazziniana (Lizer, 1917) (Hemiptera, Psyllidae), inseto conhecido vulgarmente pelos nomes de "ampola" ou "ampola-da-erva-mate", tida como praga "secundária" até recentemente, passou a causar danos de importância econômica nos ervais do Estado de Santa Catarina (CHIARADIA et al., 2000). Na Argentina, este psilídeo é a principal praga da erva-mate e os danos que ela causa provocam perdas de até $35 \%$ da produção (DIAZ, 1997).

A praga ataca erveiras de todas as idades, desde mudas até árvores adultas. Este psilídeo, ao sugar a seiva nas brotações, provoca a formação de estruturas semelhantes a "ampolas" ou "cartuchos" nas folhas novas, que abrigam as ninfas até os indivíduos tornarem-se adultos. As folhas deformadas geralmente caem após a saída dos insetos, reduzindo a produtividade dos ervais (PENTEADO, 1995; CHIARADIA et al., 2000).

Estudos conduzidos com a "ampola-daerva-mate" em laboratório, em condições controladas de temperatura $\left(25^{\circ} \mathrm{C}\right)$, umidade relativa do ar $(70 \pm 10 \%)$ e fotofase (14 horas), revelaram que o ciclo biológico deste inseto teve duração de $27,29 \pm 0,18$ dias. O período de pré-oviposição foi de 4,41 $\pm 0,23$ dias, tendo cada fêmea realizado em média duas posturas, com $22,97 \pm 1,96$ ovos, com viabilidade média de $52,28 \pm 0,23 \%$. Os estágios ninfais apresentaram quatro ou cinco instares. A razão sexual (fêmeas/total de indivíduos) foi de 0,52 e a longevidade média das fêmeas e machos foi de $9,23 \pm 0,98$ e $11,03 \pm 1,23$ dias, respectivamente
(SABEDOT et al., 1999). No levantamento populacional deste psilídeo no campo, a razão sexual foi de 0,43 (ZIDKO, 1998).

CHIARADIA \& MILANEZ (1997), estudando a atratividade da "ampola-da-erva-mate" por armadilhas de diferentes cores, concluíram que bandejas de cor vermelha são adequadas para o seu monitoramento. Estas bandejas devem ser instaladas entre as filas de árvores, sobre suportes de madeira com $1,40 \mathrm{~m}$ de altura. Elas devem conter água e detergente para que os insetos atraídos sejam retidos. O monitoramento desta praga poderá ser conduzido também com armadilhas luminosas, equipadas com lâmpada ultravioleta, modelo F15 T12 (CHIARADIA et al., 2000).

Algumas informações sobre aspectos da ecologia da $\boldsymbol{G}$. spegazziniana são contraditórias. Assim, MATTOS (1982) comenta que a maior infestação deste inseto ocorre no período de novembro a fevereiro. GALLO et al. (1988) citam que o pico de infestação ocorre no período de setembro a novembro, enquanto ZIDKO (1998) constatou a ocorrência das maiores infestações nos meses de fevereiro, maio e outubro, épocas que coincidem com os principais períodos de brotação das erveiras a região de Chapecó. Na Argentina, os períodos de maior ataque deste psilídeo são de março a maio e de agosto a outubro (FLORES \& NOTTA, s.d.).

A flutuação de cigarrinhas de pastagens foi estudada por MILANEZ et al. (1983), através de levantamentos quinzenais das populações dos insetos no campo. Estudo de regressão linear entre o número de insetos capturados e dados mensais de temperatura mínima geraram uma equação que, quando foi igualada a zero, permitiu estimar a temperatura base ou limiar para as cigarrinhas, possibilitando identificar as constantes térmicas em graus-dia e os números das prováveis gerações anuais para Zulia entreriana (Berg., 1879) e Deois flavopicta (Stal, 1854) (Hemiptera, Cercopidae).

LARA et al. (1977), investigando as influências de fatores climáticos sobre a população de diversas pragas de citros e de seus inimigos naturais, verificaram que o surgimento e os picos de infestação do "pulgão-preto"- Toxoptera citricidus (Kirk., 1907) (Hemiptera, Aphididae) estava associado com a brotação das árvores.

Muitos autores constataram a existência de influências dos fatores climáticos sobre a flutuação populacional de insetos. SILVEIRA NETO et al. (1976) cita que as maiores infestações da "broca-da-figueira" - Azochis gripusalis Walker, 1859 (Lepidoptera, Pyralidae) são verificadas nos meses de temperatura mais elevada. Segundo LARA 
et al. (1977), a variação populacional da Diabrotica speciosa (Germar, 1824) (Coleoptera, Chrysomelidae) apresenta correlação positiva com as variações da temperatura mínima, temperatura máxima e insolação e correlação negativa com a velocidade dos ventos; enquanto a flutuação da "mosca-do-mediterrâneo" Ceratitis capitata (Wied., 1824) (Diptera, Tephritidae) mostrou correlação positiva com temperatura máxima e negativa com a umidade relativa do ar. MILANEZ et al. (1983) relata sobre a existência de correlação positiva entre a temperatura mínima e a população de cigarrinhas das pastagens.

Esta pesquisa teve por objetivo estudar aspectos da ecologia de G. spegazzinianna e identificar as interferências dos fatores ambientais sobre sua flutuação populacional, visando obter subsídios capazes de implementar o Manejo Integrado desta praga.

\section{MATERIAL E MÉTODOS}

O levantamento populacional de $\boldsymbol{G}$. spegazziniana foi realizado no distrito de Marechal Bormann, município de Chapecó, em dois ervais da Empresa Ouro Verde, situados na latitude $27^{\circ} 11^{\prime}$ Sul, longitude $52^{\circ} 39^{\prime}$ Oeste e com altitude média de $675 \mathrm{~m}$. As árvores, conduzidas no espaçamento de $1,50 \times 2,50 \mathrm{~m}$, possuíam oito anos de idade e troncos com a altura média de poda de $1,40 \mathrm{~m}$.

As amostragens foram realizadas no período de maio de 1997 a abril de 2000, utilizandose quatro bandejas por erval, conforme metodologia descrita por CHIARADIA \& MILANEZ (1997). As amostras foram recolhidas 48 horas após a instalação das armadilhas, transportadas para o Laboratório de Fitossanidade da Epagri-CPPP (Empresa de Pesquisa Agropecuária e Extensão Rural de Santa Catarina - Centro de Pesquisa para Pequenas Propriedades), onde os insetos foram contados e sexados com o auxílio de microscópio estereoscópio de 40 aumentos.

Os dados mensais de adultos de $\boldsymbol{G}$. spegazziniana capturados no período de maio de 1997 a abril de 2000 foram utilizados nos estudos de razão sexual da espécie. Os insetos capturados no período de maio de 1998 a abril de 2000 foram correlacionados com as médias mensais de temperaturas mínimas (Tmi), em ${ }^{\circ} \mathrm{C}$; total mensal de precipitação pluviométrica (p), em $\mathrm{mm}$; total mensal de horas de insolação (i); umidade relativa do ar (UR); e velocidade dos ventos (v), em $\mathrm{m} / \mathrm{s}$; dados coletados na Estação Meteorológica da Epagri-CPPP. Análise de regressão foi procedida entre o número mensal de psilídeos capturados e as médias mensais de temperaturas mínimas, sendo a temperatura base (Tb) estimada ao igualar a equação resultante à zero.

A constante térmica $(\mathrm{K})$ em graus-dia (GD) para a G. spegazziniana foi calculada pela fórmula de Reaumur, citada por SILVEIRA NETO et al. (1976), expressa por $\mathrm{K}=\mathrm{D}(\mathrm{T}-\mathrm{Tb})$; na qual "D" caracteriza os dias necessários para a "ampolada-erva-mate" completar seu ciclo biológico e " $T$ " representa a temperatura que foi utilizada no estudo. Para o cálculo anual de graus-dia, foi utilizado o procedimento proposto por ARNOLD (1960), apud MILANEZ et al. (1983), que se constitui do somatório anual das médias mensais de temperaturas mínimas, diminuídas da temperatura base e multiplicadas pelo número de dias de cada mês, nos meses que a Tmi > Tb. O número provável de gerações anuais de G. spegazziniana foi estimado pela divisão do somatório anual médio de graus-dia pela sua constante térmica $(\mathrm{K})$.

\section{RESULTADOS E DISCUSSÃO}

O número de espécimes adultos de $\boldsymbol{G}$. spegazziniana capturados no período de maio de 1997 a abril de 2000 alcançou 21.044 exemplares, sendo 12.064 machos e 8.980 fêmeas, que resultou na razão sexual de 0,42 (Tabela 1 ). Este resultado está em conformidade ao obtido por ZIDKO (1998), que constatou razão sexual de 0,43 em seus levantamentos de campo. A razão sexual de 0,52, obtida para esta espécie nos estudos conduzidos por SABEDOT et al. (1999) em laboratório, pode ser explicada pelas contagens terem considerado apenas o sexo dos insetos, sem considerar a sua longevidade, haja vista que, nas avaliações de campo, o número de machos tende a ser superior

Tabela 1 - Número de adultos de Gyropsylla spegazziniana capturados em dois ervais, durante a condução do experimento, no período de maio de 1997 a abril de 2000. Chapecó, SC

\begin{tabular}{|c|c|c|c|c|c|c|c|}
\hline & \multicolumn{3}{|c|}{ LOCAL 1} & \multicolumn{3}{|c|}{ LOCAL 2} & \multirow{2}{*}{$\begin{array}{l}\text { TOTAL } \\
\text { GERAL }\end{array}$} \\
\hline & Machos & Fêmeas & Total & Machos & Fêmeas & Total & \\
\hline $\begin{array}{l}\text { INSETOS } \\
\text { COLETADOS }\end{array}$ & 5.854 & 4.615 & 10.469 & 6.210 & 4.365 & 10.575 & 21.044 \\
\hline $\begin{array}{l}\text { TOTAL POR } \\
\text { SEXO }\end{array}$ & \multicolumn{3}{|c|}{12.064 machos } & \multicolumn{3}{|c|}{8.980 machos } & \\
\hline
\end{tabular}

Ciência Rural, v. 32, n. 3, 2002. 
devido a sua maior longevidade em relação às fêmeas.

A elevada infestação e os expressivos danos provocados pela "ampola-da-erva-mate" nos ervais da região Oeste Catarinense permitem enquadrar este psilídeo como uma das pragas "chave" da cultura da erva-mate. $\mathrm{O}$ número de indivíduos capturados nas avaliações deste experimento (Tabela 2) caracteriza a elevada incidência desta praga na região.

A distribuição de adultos de $\boldsymbol{G}$. spegazziniana nos ervais que, pela classificação de BEGON et al. (1987), ocorre de forma agregada e/ou aleatória, geralmente está associada à presença de brotações nas árvores, condição necessária para que os insetos façam suas posturas. Este comportamento da praga pode provocar variação no número de insetos capturados em diferentes locais e/ou datas de amostragens, sendo necessário observar as condições fenológicas das planta, por ocasião do monitoramento das infestações. Estas variações podem ser observadas neste experimento, conforme expresso na tabela 2.

Tabela 2 - Adultos de Gyropsylla spegazziniana capturados mensalmente em dois ervais no período maio de 1998 a abril de 2000, médias mensais das temperaturas mínimas (Tmi), máximas (Tma) e médias (Tme), graus-dia (GD) e graus-dia/mês.Chapecó, $\mathrm{SC}$.

\begin{tabular}{|c|c|c|c|c|c|c|c|c|c|}
\hline \multirow{2}{*}{ Ano } & \multirow{2}{*}{ Mês } & \multicolumn{3}{|c|}{ Número de "ampolas" } & \multicolumn{3}{|c|}{ Temperatura $\left({ }^{\circ} \mathrm{C}\right)$} & \multirow{2}{*}{$\begin{array}{c}\text { Graus- } \\
\text { dia }\end{array}$} & \multirow{2}{*}{$\begin{array}{l}\text { Graus- } \\
\text { dia/mês }\end{array}$} \\
\hline & & Local 1 & Local 2 & Total & Tmi & Tma & Tme & & \\
\hline \multirow[t]{8}{*}{1998} & Maio & 9 & 9 & 18 & 11,9 & 20,7 & 16,30 & 05,94 & 184,18 \\
\hline & Junho & 2 & 3 & 5 & 09,6 & 20,0 & 14,80 & -- & --- \\
\hline & Julho & 8 & 12 & 20 & 11,5 & 21,6 & 16,55 & 06,19 & 191,89 \\
\hline & Agosto & 4 & 49 & 53 & 12,4 & 20,5 & 16,45 & 06,09 & 188,79 \\
\hline & Setembro & 6 & 29 & 35 & 12,4 & 21,6 & 17,00 & 06,64 & 199,20 \\
\hline & Outubro & 88 & 343 & 431 & 14,9 & 25,5 & 20,20 & 09,84 & 305,04 \\
\hline & Novembro & 403 & 731 & 1.134 & 16,1 & 28,5 & 22,30 & 11,94 & 358,20 \\
\hline & Dezembro & 1.776 & 1.576 & 3.352 & 17,2 & 27,9 & 22,55 & 12,19 & 377,89 \\
\hline \multirow[t]{13}{*}{1999} & Janeiro & 435 & 1.429 & 1.864 & 18,1 & 29,7 & 23,90 & 13,54 & 419,74 \\
\hline & Fevereiro & 201 & 509 & 710 & 18,8 & 29,0 & 23,90 & 13,54 & 379,12 \\
\hline & Março & 301 & 277 & 578 & 18,9 & 30,1 & 24,50 & 14,14 & 438,34 \\
\hline & Abril & 291 & 168 & 459 & 14,5 & 24,3 & 19,40 & 09,04 & 271,20 \\
\hline & \multicolumn{7}{|c|}{ Sub total } & & $3.313,99$ \\
\hline & Maio & 82 & 93 & 175 & 10,4 & 20,9 & 15,65 & 05,29 & 163,99 \\
\hline & Junho & 18 & 24 & 42 & 09,7 & 18,8 & 14,25 & --- & --- \\
\hline & Julho & 28 & 24 & 52 & 09,9 & 19,3 & 14,60 & --- & --- \\
\hline & Agosto & 53 & 72 & 125 & 10,8 & 23,6 & 17,20 & 06,84 & 212,04 \\
\hline & Setembro & 35 & 41 & 76 & 13,3 & 25,3 & 19,30 & 08,94 & 268,20 \\
\hline & Outubro & 69 & 324 & 393 & 13,5 & 23,9 & 18,70 & 08,34 & 258,54 \\
\hline & Novembro & 84 & 134 & 218 & 14,3 & 26,6 & 20,45 & 10,09 & 302,70 \\
\hline & Dezembro & 66 & 1.151 & 1.217 & 18,0 & 29,0 & 23,50 & 13,14 & 407,34 \\
\hline \multirow[t]{5}{*}{2000} & Janeiro & 660 & 156 & 816 & 19,0 & 30,0 & 24,50 & 14,14 & 438,34 \\
\hline & Fevereiro & 95 & 486 & 581 & 18,5 & 28,9 & 23,70 & 13,34 & 373,52 \\
\hline & Março & 1.022 & 696 & 1.718 & 17,4 & 28,2 & 22,80 & 12,44 & 358,64 \\
\hline & Abril & 892 & 321 & 1.213 & 15,5 & 26,2 & 20,85 & 10,49 & 314,70 \\
\hline & \multicolumn{7}{|c|}{ Subtotal } & & $3.125,01$ \\
\hline Média & & & & & & & & & $3.219,30$ \\
\hline
\end{tabular}

Os dados mensais de adultos de $\boldsymbol{G}$. spegazziniana capturados nas armadilhas no período de maio de 1998 a abril de 2000, ao serem correlacionados com os dados das seguintes variáveis climáticas: médias mensais de temperaturas mínimas (Tmi), em ${ }^{\circ} \mathrm{C}$; total mensal de horas de insolação (i); total mensal de precipitação pluviométrica (p), em $\mathrm{mm}$; umidade relativa do ar (UR); e velocidade dos ventos (v), em $\mathrm{m} / \mathrm{s}$; expressou valores de "r" de 0,$63 ; 0,60 ; 0,02 ;-0,27$; e - 0,31, respectivamente. Os deslocamentos das linhas das figuras 1 e 2 mostram as variações do número de psilídeos capturados nas armadilhas e os dados das médias mensais de temperatura mínima e insolação verificados no período do estudo, respectivamente. Os dados referentes aos insetos capturados de maio de 1997 a abril de 1998 só foram considerados nos estudos de razão sexual, devido à ocorrência de condições climáticas atípicas, além de terem sido realizadas podas nos ervais, em épocas que poderiam interferir nos resultados das análises de correlação e regressão linear.

Para verificar a influência dos fatores climáticos sobre a flutuação populacional deste inseto, foram conduzidos estudos de análise de regressão linear, que resultou na equação $\hat{y}=-1621,47+156,37$ Tmi, com coeficiente de determinação $r^{2}=0,40$ que, apesar de ser um valor pouco expressivo numericamente, caracteriza a tendência do comportamento da espécie. As outras variáveis estudadas não apresentaram resultados significativos, quando submetidas ao teste " $F$ ". O menor número de insetos capturados nos meses que tiveram as menores temperaturas mínimas (Tabela 2) sugere que temperaturas baixas são limitantes para o desenvolvimento desta espécie. Pelo fato da média mensal de temperatura mínima ter apresentado resposta na variação do número de psilídeos capturados, a temperatura "base" (Tb) para G. spegazziniana foi estimada ao atribuir valor zero para a equação resultante da análise de regressão linear. Assim, o resultado de $10,36^{\circ} \mathrm{C}$

Ciência Rural, v. 32, n. 3, 2002. 


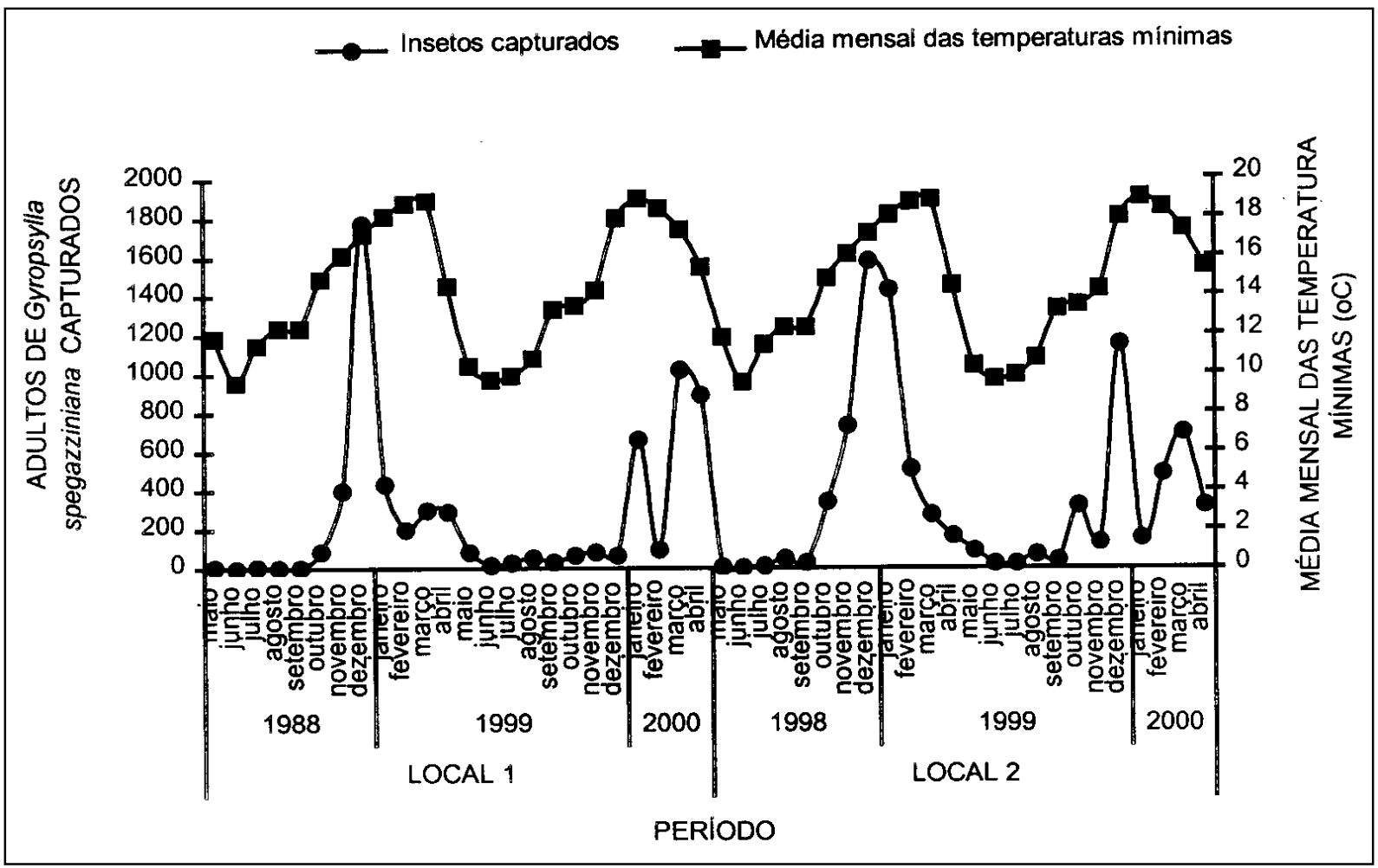

Figura 1 - Flutuação populacional de adultos de Gyropsylla spegazziniana capturados em dois ervais, no período de maio de 1998 a abril de 2000 e médias mensais de temperaturas mínimas. Chapecó, SC.

expressa o limite inferior de temperatura para o desenvolvimento da "ampola-da-erva-mate". O fato de a região de Chapecó apresentar médias mensais de temperaturas mínimas geralmente superiores a $10,36^{\circ} \mathrm{C}$ caracteriza boa adaptação desta espécie ao meio. Assim, foi possível verificar que, nos meses em que a temperatura mínima ficou próxima da temperatura base $(\mathrm{Tb})$, houve pequena variação na população deste inseto, enquanto nos meses em que a média mensal de temperatura mínima foi mais elevada, os níveis de infestação aumentaram.

A constante térmica $(\mathrm{K})$ em graus-dia para a $\boldsymbol{G}$. spegazziniana foi calculada através da fórmula $\mathrm{K}=\mathrm{D}(\mathrm{T}-\mathrm{Tb})$; onde " $\mathrm{D}$ " expressa os 27,29 dias necessários para "ampola-da-erva-mate" completar seu ciclo biológico e "T" $=25^{\circ} \mathrm{C}$, temperatura na qual foram conduzidos os estudos da biologia deste inseto por SABEDOT et al. (1999). Assim, o resultado de $\mathrm{K}=399,52$ graus-dia caracteriza a necessidade térmica para a "ampola" completar seu ciclo biológico.

A estimativa do total anual de graus-dia para G. spegazziniana foi calculado pela soma graus-dia mensais, diminuídos da temperatura base $\left(10,36^{\circ} \mathrm{C}\right)$ e os resultados multiplicados pelo número de dias de cada mês. Este cálculo foi procedido para os meses que apresentaram a média mensal de temperatura mínima maior que a temperatura base (Tabela 2). A média anual de 3.219,30 graus-dia foi obtida pela média do somatório dos graus-dia verificados nos períodos de maio de 1998 a abril de 1999 e de maio de 1999 a abril de 2000, que foram de $3.313,99$ e $3.125,01$. A divisão da média anual de graus-dia pela constante térmica da $\boldsymbol{G}$. spegazziniana, resultou em 8,05 possíveis gerações anuais da praga na região de Chapecó.

\section{CONCLUSÕES}

Nas condições em que o experimento foi conduzido e pelos resultados obtidos é possível concluir que a temperatura mínima exerce influência sobre a população de $\boldsymbol{G}$. spegazzinianna, sendo sua temperatura base de $10,36^{\circ} \mathrm{C}$. Também se pôde verificar que são necessários 399,52 graus-dia para a $\boldsymbol{G}$. spegazzinianna completar seu ciclo biológico e que podem ocorrer até 8,05 gerações anuais da “ampola-da-erva-mate” na região de Chapecó-SC. 


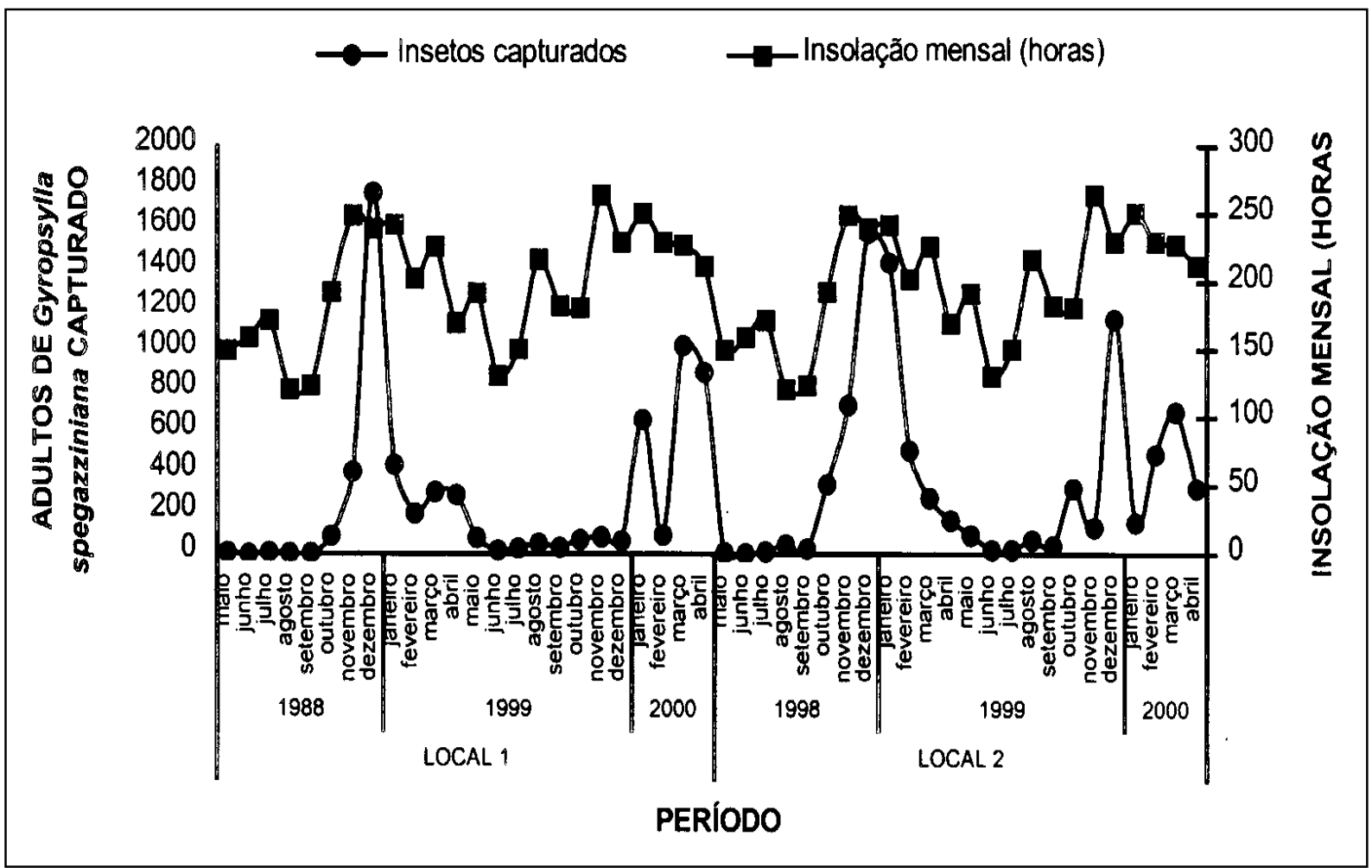

Figura 2 - Flutuação populacional de adultos de Gyropsylla spegazziniana capturados em dois ervais, no período de maio de 1998 a abril de 2000 e total de horas mensais de insolação. Chapecó, SC

\section{AGRADECIMENTOS}

Agradecemos à Empresa Ouro Verde pela disponibilidade dos ervais utilizados na condução dos estudos; aos Engenheiros Agrônomos da Epagri: Renato Dietrich e Telmo Canton, pela realização e interpretações das análises estatísticas; e aos alunos da UNOESC-Campus de Chapecó, estagiários e/ou bolsistas da Epagri-CPPP: Luiz César Souza, Josiane Arsego, Janaina Meister, Cristiano R. Lajus e Josiane Cortina, pelo auxílio nas avaliações do experimento e triagem dos insetos.

\section{REFERÊNCIAS BIBLIOGRÁFICAS}

ANUÁRIO BRASILEIRO DA ERVA-MATE 2000. Santa Cruz do Sul : Gazeta Grupo de Comunicações, 2000. 80p.

BEGON, M., HARPER, J.L., TOWNSEND, C.R. Ecologia: individuos, poblaciones $y$ comunidades. Barcelona Omega, 1987. 360p.

CHIARADIA, L.A., MILANEZ, J.M. Atratividade de armadilhas coloridas a Gyropsylla spegazziniana (Lizer, 1917) (Homoptera, Psyllidae). Pesquisa Agropecuária Gaúcha, Porto Alegre, v.3, n.2, p.183 -185, 1997.

CHIARADIA, L.A., MILANEZ, J.M., SABEDOT, S.M Caracterização e danos da ampola-da-erva-mate. Revista Agropecuária Catarinense, Florianópolis, v.13, n.1, p.50$53,2000$.

DA CROCE, D.M., FLOSS, P.A. Cultura da erva-mate em Santa Catarina. Florianópolis : Epagri, 1999. 81p. (Boletim Técnico, 100).
DIAZ, C.Y.F. Perspectivas del manejo integrado de plagas em yerba mate. In: REUNIÃO TÉCNICA DO CONE SUL SOBRE A CULTURA DA ERVA-MATE, 2, Curitiba, PR 1997. Anais... Curitiba : EMBRAPA-CNPF, 1997. p.371390. (Documento, 33)

FLORES, S.R., NOTTA, D. El rulo de la yerba mate. Cerro Azul : INTA, s.d. 10p.

GALlO, D., NAKANO, O., SILVEIRA NETO, S., $\boldsymbol{e} \boldsymbol{t} \boldsymbol{a}$. Manual de entomologia agrícola. 2.ed. Piracicaba : Ceres, 1988. 649p.

LARA, F.M., BORTOLI, S.A. de, OLIVEIRA, E.A. Flutuações populacionais de alguns insetos associados ao Citrus sp. e suas correlações com fatores meteorológicos. Científica, Jaboticabal, v.5, n.2, p.134-143, 1977.

MATTOS, N.F. Estudos preliminares sobre pragas da ervamate. Porto Alegre: IPRNR, 1982. 18p.

MILANEZ, J.M., MILDE, L.C.E., PARRA, J.R.P. Estimativa da constante térmica das cigarrinhas das pastagens Zulia (Notozulia) entreriana (Berg, 1897) e Deois (Acanthodeois) flavopicta (Stal, 1854) (Homoptera: Cercopidae). Anais da Sociedade Entomológica do Brasil, v.12, n.2, p.151-163, 1983

PENTEADO, S. do R. C. Principais pragas da erva-mate e medidas alternativas para o seu controle. In: WINGE, H., FERREIRA, A.G.; MARIATH, J.E., et al. Erva-mate: biologia e controle no Cone sul. Porto Alegre : UFRGS, 1995. 356p. 
SABEDOT, S.M., MILANEZ, J.M., GARCIA, F.R.M. et al. Biologia de Gyropsylla spegazziniana (Lizer, 1917) (Homoptera: Psyllidae) em laboratório. Acta Biológica Leopoldensia, São Leopoldo, v.21, n.2. p.203-212, 1999.

SILVEIRA NETO, S., NAKANO, O., BARDIN, D., et al. Manual de ecologia de insetos. Piracicaba : Ceres, 1976. 419p.
ZIDKO, A. Flutuação populacional de Gyropsylla spegazziniana (Lizer, 1917) (Homoptera: Psyllidae) no Município de Chapecó. Chapecó SC, 1988. 44p. Monografia (Graduação) - Curso de Graduação em Biologia, Universidade do Oeste de Santa Catarina, Campus de Chapecó, 1998.

Ciência Rural, v. 32, n. 3, 2002. 